\title{
Discrimination of Schroeder-Phase Harmonic Complexes by Normal-Hearing and Cochlear-Implant Listeners
}

\author{
Ward R. Drennan, ${ }^{1}$ Jeff K. Longnion, ${ }^{1,2}$ Chad Ruffin, ${ }^{1}$ and Jay T. Rubinstein, ${ }^{1,2}$ \\ ${ }^{1}$ Virginia Merrill Bloedel Hearing Research Center, Department of Otolaryngology, Head and Neck Surgery, University \\ of Washington, Box 357923, Seattle, WA 98195, USA \\ ${ }^{2}$ Department of Bioengineering, University of Washington, Seattle, WA 98195, USA
}

Received: 13 December 2006; Accepted: 13 November 2007; Online publication: 8 December 2007

\begin{abstract}
The temporal fine structure (TFS) of sound contributes significantly to the perception of music and speech in noise. The evaluation of new strategies to improve TFS delivery in cochlear implants (CIs) relies upon the assessment of fine structure encoding. Most modern CI sound processing schemes do not encode withinchannel TFS per se, but some TFS information is delivered through temporal envelope cues across multiple channels. Positive and negative Schroeder-phase harmonic complexes differ primarily in acoustic TFS and provide a potential test of TFS discrimination ability in CI users for current and future processing strategies. The ability to discriminate Schroeder-phase stimuli was evaluated in $24 \mathrm{CI}$ users and 7 normal-hearing listeners at four fundamental frequencies: 50, 100, 200, and $400 \mathrm{~Hz}$. The dependent variables were percent correct at each fundamental frequency, average score across all fundamental frequencies, and a maximum-likelihoodpredicted threshold fundamental frequency for $75 \%$ correct. CI listeners scored better than chance for all fundamental frequencies tested. The $50-\mathrm{Hz}$, average, and predicted threshold scores correlated significantly with consonant-nucleus-consonant word scores. The $200-\mathrm{Hz}$ score correlated with a measure of speech perception in speech-shaped noise. Pitch-direction sensitivity is predicted jointly by the $400-\mathrm{Hz}$ Schroeder score and a spectral ripple discrimination task. The results demonstrate that the Schroeder test is a poten-
\end{abstract}

Correspondence to: Ward R. Drennan - Virginia Merrill Bloedel Hearing Research Center, Department of Otolaryngology, Head and Neck Surgery - University of Washington - Box 357923, Seattle, WA 98195, USA. Telephone: +1-206-8971848; fax: +1-206-6161828; e-mail: drennan@u.washington.edu tially useful measure of clinically relevant temporal processing abilities in CI users.

Keywords: temporal processing, fine structure, phase discrimination, cochlear implant

\section{INTRODUCTION}

Speech recognition with no background noise is often quite good in cochlear implant (CI) users; however, speech recognition deteriorates significantly in the presence of background noise (Zeng 2004) and especially in fluctuating background noise (Nelson et al. 2003; Zeng et al. 2005). Additionally, music perception and localization abilities are much worse in CI users than in normal-hearing listeners (Gfeller et al. 1997, 2002, 2005; Senn et al. 2005; Nimmons et al. 2007), highlighting the need for improvement in CI technology.

Acoustic temporal fine structure (TFS) has been shown to be critical for good performance in difficult listening tasks, such as music perception (Kong et al. 2004), speech perception in fluctuating background noise (Nelson et al. 2003; Qin and Oxenham 2003; Zeng et al. 2005; Füllgrabe et al. 2006), and localization and binaural unmasking (Drennan et al. 2007). Rosen (1992) defined TFS in terms of the TFS cues that are important for speech between about 0.6 and $10 \mathrm{kHz}$. The present paper takes a physics-based view of TFS rather than a speech-based view. TFS is defined as the rapid fluctuations of sound that directly track the fluctuations in acoustic pressure. TFS can have any frequency, but frequencies relevant to normal hearing range from about $20 \mathrm{~Hz}$ to 4 to $5 \mathrm{kHz}$, above 
which the auditory nerve fibers cannot phase-lock (Johnson 1980). The "envelopes" of acoustic waves follow fluctuation of the peaks of TFS over time.

Modern CIs process stimuli into 16 or 22 narrow bands, extract the narrow-band acoustic temporal envelopes from each filter, and use these envelopes to modulate a biphasic pulsatile carrier of constant rate (Loizou 1998; Wilson 2004; Drennan and Rubinstein 2006). Thus, the pulsatile carriers bear no TFS information. However, some acoustic TFS information can still be delivered through the narrow-band electric envelopes. Given a broadband stimulus with a flat (nonfluctuating) temporal envelope, narrow-band envelope cues can be recovered via filtering (Ghitza 2001; Zeng et al. 2004; Gilbert and Lorenzi 2006). CI users are rarely able to follow temporal envelope modulations over $300 \mathrm{~Hz}$, but there are some "star" exceptions (Townshend et al. 1987; Shannon 1992; Zeng 2002). Thus, low-frequency TFS $(<300 \mathrm{~Hz})$ could be transmitted to CI users via narrow-band temporal envelopes. The degree to which acoustic TFS is transmitted to CI users via electric temporal envelope cues is much less than the degree to which TFS is delivered to normalhearing listeners, hence the difficulty with tasks that require TFS for good performance. To measure improvement in the delivery of acoustic TFS, a good test of the TFS discrimination ability of CI users is required.

Positive and negative Schroeder-phase stimuli differ only in their TFS. These stimuli are time-reversed sound pairs with identical long-term spectra and minimal envelope modulations (Schroeder 1970).

(+) Phase $50 \mathrm{~Hz}$ Complex

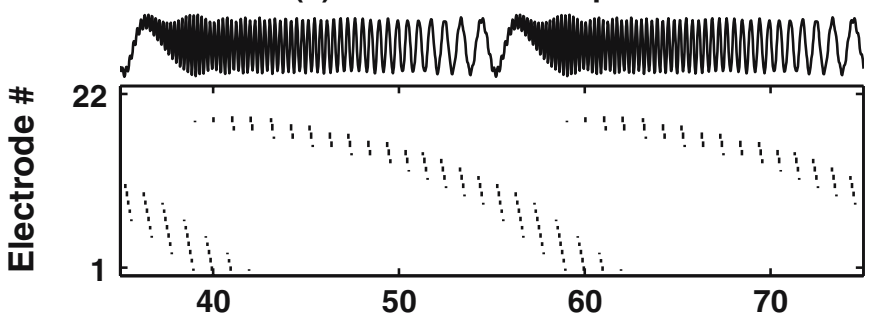

(+) Phase $400 \mathrm{~Hz}$ Complex

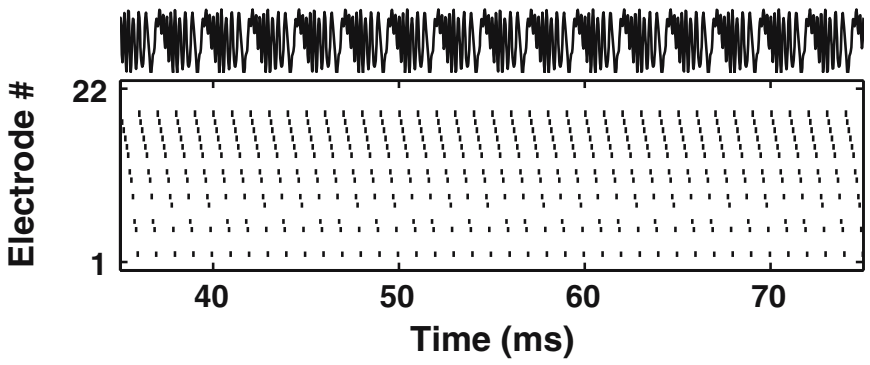

FIG. 1. Pairs of $50-\mathrm{Hz}$ (top, left and right) and $400-\mathrm{Hz}$ (bottom, left and right) Schroeder-phase complexes and their resulting electrodograms (immediately under each waveform). This electrodogram
Schroeder-phase stimuli have been used to measure sensitivity to TFS with minimal envelope cues in humans and birds (Dooling et al. 2002), as maskers to explore various phenomena in normal and hearingimpaired listeners (Kohlrausch and Sander 1995; Summers and Leek 1998; Summers 2000), and as a stimulus to explore the effect of phase on basilar membrane motion and the acoustic reflex (Summers et al. 2003; Kubli et al. 2005). Schroeder-phase harmonic complexes also appear promising for testing acoustic TFS delivery in CI users.

Figure 1 shows how the common $\mathrm{ACE}^{\circledR}$ processor encodes positive and negative Schroeder-phase stimuli with fundamental frequencies of 50 and $400 \mathrm{~Hz}$. The Schroeder-phase stimuli are transformed into sweeps of envelope packets that either rise or fall in frequency and repeat. The speed with which the sweep repeats depends on the fundamental frequency of the Schroeder-phase harmonic complex. By measuring Schroeder-phase discrimination for different fundamental frequencies, we can evaluate the ability of CI users to differentiate a rising sweep from a falling sweep as a function of the speed of the sweep. Such rapid changes in frequency occur in consonantvowel transitions in speech (Kewley-Port et al. 1983), so some correlation between speech understanding and Schroeder-phase discrimination would be expected. Furthermore, if future processing schemes encode TFS explicitly, a Schroeder-phase task could be used to measure the extent to which the processing is successful.
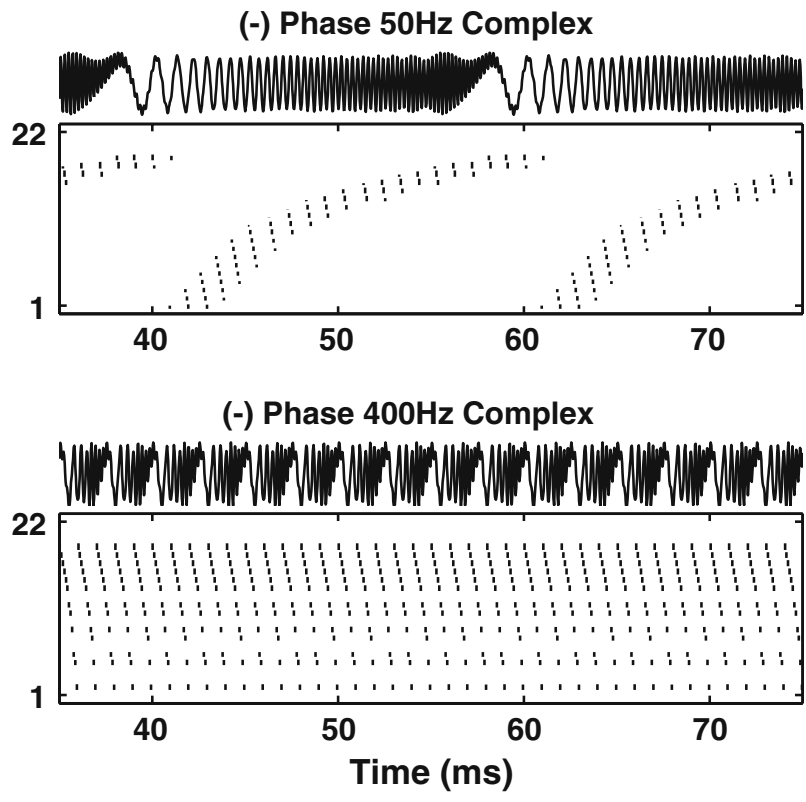

represents, as vertical lines, the biphasic pulses predicted by an $\mathrm{ACE}^{\circledR}$ signal processing strategy. Electrode number refers to the depth of insertion into the cochlea $(1=$ apical, $22=$ basal $)$. 
The first objective of the present study is to evaluate Schroeder-phase discrimination as a measure of temporal processing capabilities in CI users. The utility of the task for evaluating performance with different processing schemes can be evaluated if reliability of the test is good and learning is limited. Therefore, the tests are repeated on separate days. Another objective is to evaluate the extent to which the task is predictive of clinically relevant performance which might depend on TFS encoding. Thus, the consonant-nucleusconsonant (CNC) word test (Peterson and Lehiste 1962), a speech reception threshold (SRT) test in steady noise and two-talker babble (Turner et al. 2004; Won et al. 2007), and a clinical assessment of music perception (pitch, timbre, and isochronous melody discrimination) (Nimmons et al. 2007) were also conducted. Finally, because the TFS of the Schroederphase stimulus is transformed into a repeating frequency sweep in CI users, the Schroeder-phase test could involve both spectral and temporal discrimina- tion abilities; therefore, the extent to which Schroederphase discrimination ability is independent from a primarily spectral task is also evaluated by testing spectral ripple discrimination ability. Additionally, the combination of spectral and temporal elements in the two tasks is expected to more strongly predict clinical success.

\section{METHODS}

\section{Listeners}

A total of 24 postlingually deafened adult CI users volunteered for participation. Relevant clinical and demographic information is summarized in Table 1. CI users ranged in age from 28 to 74 , with an average of 56 years. They had an average of 4 years experience with their implants. Additionally, seven normal-hearing listeners (detection thresholds less than $20 \mathrm{~dB}$ HL at all audiometric frequencies) volunteered for participation in the study. Their ages ranged from 26 to 40, with an

\section{TABLE 1}

\begin{tabular}{|c|c|c|c|c|c|c|c|c|}
\hline \multicolumn{9}{|c|}{ Summary of demographic and clinical parameters for participating $\mathrm{Cl}$ listeners } \\
\hline Subject ID & Gender & Age & $\begin{array}{l}\text { Duration } \\
\text { of hearing }\end{array}$ & Etiology of hearing loss & $\begin{array}{l}\text { Years with } \\
\text { implant }\end{array}$ & Implant type & $\begin{array}{l}\text { Main processing } \\
\text { strategy }\end{array}$ & $\begin{array}{l}\text { CNC word } \\
\text { score }\end{array}$ \\
\hline SO000C & M & 28 & 12 & Unknown & 7 & (R) Clarion $\mathrm{Cl}$ & $\mathrm{CIS}$ & 60 \\
\hline S0010S & $\mathrm{F}$ & 76 & 5 & Otosclerosis & 0.3 & (L) HiRes90K-Auria & HiRes-S & 50 \\
\hline S0014D & $M$ & 46 & 10 & Unknown & 1 & (L) Nucleus 22 & $\mathrm{ACE}$ & \\
\hline S0015J & $\mathrm{F}$ & & & (R) Nucleus 22 & SPEAK & 82 & & \\
\hline S0016S & $M$ & 50 & 17 & Unknown & 1 & (L) HiRes90K-Auria & HiRes-S & 46 \\
\hline S0017L & $\mathrm{F}$ & 55 & 19 & Unknown & 7 & (L) Nucleus $24 \mathrm{M}$ & $\mathrm{ACE}$ & \\
\hline S0018S & $M$ & 74 & & $\begin{array}{l}\text { (R) sudden hearing } \\
\text { loss }(\mathrm{L}) \text { factory noise }\end{array}$ & 6 & (L) Nucleus 24M & ACE & 52 \\
\hline S0019B & $\mathrm{F}$ & 63 & 12 & Hereditary & 3 & (R) Nucleus24RCS & ACE & 88 \\
\hline SO020M & $\mathrm{F}$ & 53 & 10 & $\begin{array}{l}\text { Unknown, } \\
\text { possibly viral }\end{array}$ & 2 & (L) Nucleus 24RCS & ACE & 80 \\
\hline S0021D & M & 59 & 8 & Ostosclerosis & 6 & $\begin{array}{l}\text { (L) Nucleus 24RCS } \\
\text { (R) Nucleus24M }\end{array}$ & ACE & 90 \\
\hline SO022R & $\mathrm{F}$ & 70 & 8 & Unknown & 6 & (R) Nucleus24M & ACE & 80 \\
\hline SO023C & $\mathrm{F}$ & 60 & 15 & Unknown & 7 & (L) Nucleus24M & ACE & 56 \\
\hline S0024L & $\mathrm{F}$ & 41 & 4 & Hereditary & 2 & (R) Nucleus 24RCS & ACE & 82 \\
\hline S0025D & $M$ & 43 & 2 & Radiation & 1.7 & (L) N24RCS & $\mathrm{ACE}$ & 80 \\
\hline SO026N & $M$ & 55 & 11 & Unkown & 4 & (R) Nucleus 24RCS & ACE 62 & \\
\hline S0027M & $M$ & 50 & 24 & Hereditary & 4 & (R) Nucleus 24 RCS & $\mathrm{ACE}$ & 78 \\
\hline S0028M & $\mathrm{F}$ & 44 & 2 & Cogan's syndrome & 3 & $\begin{array}{l}\text { (L) Nucleus } 22 \\
\text { (R) Nucleus } 22\end{array}$ & ACE & 100 \\
\hline S0029D & $\mathrm{F}$ & 68 & 10 & Ostosclerosis & 5 & $\begin{array}{l}\text { (L) Cl24R (CS) } \\
\text { (R) Nucleus } 22\end{array}$ & $\begin{array}{l}\text { (L) ACE } \\
\text { (R) SPEAK }\end{array}$ & 58 \\
\hline S0030G & $M$ & 66 & 5 & Amikacin & 3.5 & $\begin{array}{l}\text { (L) Nucleus Freedom } \\
\text { (R) Nucleus } 22\end{array}$ & ACE & 80 \\
\hline S0031C & $\mathrm{F}$ & 55 & 1 & Hereditary & 1 & (R) Nucleus Freedom & ACE & 90 \\
\hline S0032A & M & 57 & 57 & Sensorineural at Birth & 3 & $\begin{array}{l}\text { (L) Nucleus 24M } \\
\text { (R) Nucleus Freedom }\end{array}$ & ACE & 52 \\
\hline S0033M & $\mathrm{F}$ & 57 & 15 & Unknown & 16 & $\begin{array}{l}\text { (L) Nucleus 24RCS } \\
\text { (R) Nucleus } 22\end{array}$ & $\begin{array}{l}\text { (L) CIS } \\
\text { (R) SPEAK }\end{array}$ & 70 \\
\hline S0034K & $M$ & 55 & & Noise Exposure & 1.5 & (L) HiRes90K-Auria & HiRes-S & 62 \\
\hline S0036W & $M$ & 56 & 40 & Unknown & 6 & (R) Nucleus Freedom & $\mathrm{ACE}$ & 18 \\
\hline
\end{tabular}

(L) and (R) designate left and right ears, respectively 
average of 31.3 years. Each listener participated in the study in accordance with the University of Washington Institutional Review Board.

\section{Test battery administration}

All tests were performed in a sound-attenuating booth at the Virginia Merrill Bloedel Hearing Research Center. A MATLAB (The Mathworks) graphical user interface running on a Mac G5 computer was used to present sound stimuli to listeners via a Crown D45 amplifier and a free-standing speaker (B\&W DM303) placed at head level, $1 \mathrm{~m}$ in front of the listeners. CI users were instructed to turn off any hearing aids and set the loudness of their implant processor to a comfortable level. Listeners performed the task using a mouse and computer screen placed to the right of the speaker. The speaker was a studio monitor and was selected for its good amplitude and phase response. The speaker exceeded ANSI standards for speech audiometry, varying $\pm 2 \mathrm{~dB}$ from 100 to $20,000 \mathrm{~Hz}$. The phase response of these speakers was smooth across frequencies, varying \pm 30 degrees from 150 to $20,000 \mathrm{~Hz}$ and \pm 45 degrees below $150 \mathrm{~Hz}$. Normal-hearing listeners were tested only on the Schroeder-phase discrimination test, whereas CI users were administered the Schroeder-phase discrimination test as part of a battery of psychophysical tasks. In addition to the Schroeder-phase discrimination test, the test battery included CNC monosyllabic word score presented at $62 \mathrm{dBA}$ (Peterson and Lehiste 1962); a test of spectral ripple resolution (Henry and Turner 2003; Henry et al. 2005; Won et al. 2007); an assessment of speech-in-noise perception that evaluated the spondee reception threshold (SRT) (Turner et al. 2004; Won et al. 2007); and a musical test battery including a test of pitch-direction discrimination, isochronous melody identification, and timbre recognition (Nimmons et al. 2007). The order of test administration was varied, but the Schroeder-phase discrimination test was typically administered after 30 to $90 \mathrm{~min}$ of testing with other tasks. When time permitted and users were willing to undergo further testing on either the subsequent day or within a few weeks, they were retested.

The spectral ripple test, similar to the test used by Henry et al. (2005), was administered using methods and stimuli described in Won et al. (2007). The spectral ripple test measures the just noticeable difference in spectral ripple density. The stimuli had a bandwidth of $100-5,000 \mathrm{~Hz}$ and sinusoidal spectral ripples with a peak-to-valley ratio of $30 \mathrm{~dB}$. A two-up, one-down adaptive tracking procedure was used in which the density of the spectral ripples was tracked. The dependent variable was ripples per octave. Twenty-two of the $24 \mathrm{CI}$ users completed this test.

The SRT test was a closed-set task where users were asked to identify one of 12 spondees they heard in the presence of either steady-state, speech-shaped noise, or two-talker babble (Won et al. 2007). The SRT test measures the level of noise required to just mask a closed set of spondees. The level of the noise was tracked using a one-down, one-up procedure and 2-dB steps. The dependent variable was the signal-to-noise ratio. The spondee speaker was female. Twenty-two of the 24 listeners completed this test.

The "clinical assessment of music perception" (CAMP) was used for music testing (Nimmons et al. 2007). The CAMP consists of melody, pitch, and timbre discrimination tasks. The details of stimulus creation, the melodies used, and implementation of all three music tests are described in Nimmons et al. (2007). Twenty-two of the 24 listeners completed the music tests. All stimuli were presented at $65 \mathrm{dBA}$. Each music test had a short training period in which listeners could hear the melodies or instruments or practice a few pitch discrimination trials with feedback. Subsequently, during testing, no feedback was given. The melody identification test required users to identify isochronous melodies from a closed set of 12 popular melodies. Rhythm cues were removed such that notes of a longer duration were repeated in an eighth-note pattern. The test used synthesized pianolike tones with identical envelopes. A total percent correct score was calculated after 36 melody presentations, including 3 presentations of each melody.

The pitch-direction discrimination task was a twoalternative forced-choice (2AFC) task administered using the same synthesized complex, piano-like tones, and required users to identify which of two complex tones had the higher fundamental frequency. The dependent variable was the just-noticeable-difference threshold in semitones determined using a one-up, one-down tracking procedure converging on 50\% correct (Levitt 1971). Complex tones of four fundamental frequencies $(185,262,330$, and $392 \mathrm{~Hz})$ were used in the task and the primary dependent variable was the average of the threshold in semitones for all fundamental frequencies. The timbre recognition task required listeners to identify instruments from a closed set of eight instruments that played an identical melodic contour. Listeners discriminated live recordings of instruments with moderate, uniform tempo playing a simple five-note pattern. The instruments were the piano, trumpet, clarinet, saxophone, flute, violin, cello, and guitar. A total percent correct score was calculated after 24 presentations including 3 presentations of each instrument.

\section{Schroeder-phase discrimination test procedure}

A single administration of this task involved one short training block and six test blocks of trials. For each trial, a four-interval 2AFC paradigm was used. The 
training block contained only eight trials, two for each fundamental frequency $(50,100,200$, and $400 \mathrm{~Hz})$. In each test block, a total of 96 randomly ordered trials were presented, with each of the four fundamental frequencies presented 24 times. Four 500-ms stimuli were presented interleaved with $100 \mathrm{~ms}$ of silence, including three presentations of the negative Schroeder-phase stimuli and one positive-phase stimulus that randomly occurred in either the second or third interval. Listeners were asked to identify the sound that was different by choosing either the second or third interval. Visual feedback of the correct answer was given after each presentation. As described in the stimuli section, each of the four successive stimuli had a random starting phase to eliminate the onset and the offset as reliable discrimination cues. Stimuli were presented at $65 \mathrm{dBA}$ at head level $1 \mathrm{~m}$ from the speaker, and the levels of the stimuli were not roved. The percent correct was calculated for each block and fundamental frequency.

\section{Schroeder-phase stimuli}

Positive and negative Schroeder-phase stimuli pairs were created for each of four distinct fundamental frequencies. Fundamental frequencies of 50, 100, 200, and $400 \mathrm{~Hz}$ were chosen to facilitate comparison with Dooling et al.'s results (Dooling et al. 2002) and because preliminary tests suggested that these fundamental frequencies encompassed the performance range of a typical CI user. For each fundamental frequency, equal-amplitude cosine harmonics from the fundamental frequency up to $5 \mathrm{kHz}$ were summed. Each harmonic is given a phase according to the following equation:

$$
\theta_{n}= \pm \pi n(n+1) / N
$$

where $\theta_{n}$ is the phase of the $n$th harmonic, $n$ is the $n$th harmonic, $N$ is the total number of harmonics in the complex, and the positive or negative sign is used when constructing the positive or negative Schroederphase signals, respectively. During each presentation of stimuli, these harmonic complexes were randomly shifted in global phase and multiplied by a $500-\mathrm{ms}$ constant-amplitude window with a 10-ms linear onset and offset. This was done so that the portion of the harmonic complex that occurred during onset and offset could not be used as a reliable cue for discriminating pairs of complexes.

\section{Analysis}

For each fundamental frequency, the scores in each of six blocks were averaged to create an estimate of percent correct for that fundamental frequency (hereafter referred to as the 50-, 100-, 200-, and 400-Hz scores).
Additionally, an overall average percent correct (AVG) score was constructed by averaging the scores from each of four fundamental frequencies and all six blocks. Finally, a 75\%-maximum-likelihood threshold fundamental frequency (hereafter referred to as MLTF) was determined using a maximum-likelihood fit of a psychometric function as described below.

The difference between normal-hearing listeners and CI users was analyzed using a four-fundamentalfrequency by six-repetition, repeated-measures ANOVA. In addition, for each of the six candidate measures of performance $(50-\mathrm{Hz}, 100-\mathrm{Hz}, 200-\mathrm{Hz}, 400-\mathrm{Hz}, \mathrm{AVG}$, and MLTF), a nonparametric one-way ANOVA was used to assess any significant differences between the two groups. Within-test learning on the test was assessed using the $4 \times 6$ repeated-measures ANOVA. Test-retest reliability (for the subset of $18 \mathrm{CI}$ users who retook the full six-block test) was assessed using a nonparametric Wilcoxon sign-rank test. Correlation of the Schroederphase discrimination test with the other tests in the battery described above was assessed using both a Pearson's linear correlation and Spearman's rank correlation coefficient. Multiple regression and partial correlation was applied to determine if the combination of the Schroeder-phase discrimination test scores and spectral ripple scores could predict performance on speech and music perception tests better than any of them alone. Adjusted $R^{2}$ values were calculated for the multiple regression, and only regressions with significant coefficients were reported. Partial correlations controlling for the effect of the spectral ripple test were reported if Pearson correlation coefficients were significant before and after control.

Visual inspection of these and previous results (Dooling et al. 2002) suggests a relationship between fundamental frequency and percent correct discrimination that might follow a monotonic, sigmoid function typical of psychometric functions. Thus, threshold fundamental frequency could be an appropriate measure of performance on the test. A maximum-likelihood fit of a 2AFC psychometric curve was created for the data from each listener according to methods described by Madigan and Williams (1987). Because the trend was for performance to worsen as fundamental frequency increased, and because stimulus frequencies were logarithmically spaced, the negative log of the fundamental frequency was chosen as the independent variable and percent correct discrimination was chosen as the dependent variable. A custom MATLAB script was created, which found the parameters $\mu$ (mean) and $\sigma$ (standard deviation) needed to describe a psychometric curve by varying these parameters and minimizing the negative $\log$ likelihood of the data for each listener. Minimizing the negative log of the likelihood is equivalent to maximizing the likelihood. Then, a 
profile-likelihood-based 95\% confidence interval was obtained for the estimate $\mu$ (described in Venzon and Moolgavkar 1988). The mean and its confidence interval were converted back to representations of frequency, and, thus, an estimate of the $75 \%$ threshold point was obtained. When the maximum-likelihood fit predicted a threshold outside the range of $50-400 \mathrm{~Hz}$, the confidence intervals were prohibitively large. These thresholds were reported as less than $50 \mathrm{~Hz}$ or more than $400 \mathrm{~Hz}$. If, for example, a listener never reached $75 \%$ correct at any fundamental frequency, the MLTF was reported as less than $50 \mathrm{~Hz}$. This derived threshold is valid as long as a monotonic relationship exists between fundamental frequency and performance, which was the trend seen in almost all CI users tested.

\section{RESULTS}

A total of 24 CI users and 7 normal-hearing listeners completed the Schroeder-phase discrimination test. The full test took between 40 and $50 \mathrm{~min}$, allowing listeners breaks in between the six blocks of the test. Figure 2 demonstrates how the six measures of performance on the test were obtained. For each fundamental frequency, the six test blocks were averaged to obtain an AVG score. The overall performance on the test, designated as the AVG score, was obtained by averaging across all fundamental frequencies. Lastly, the scores on each block and fundamental frequency were used to fit a maximumlikelihood psychometric curve to the data, allowing a

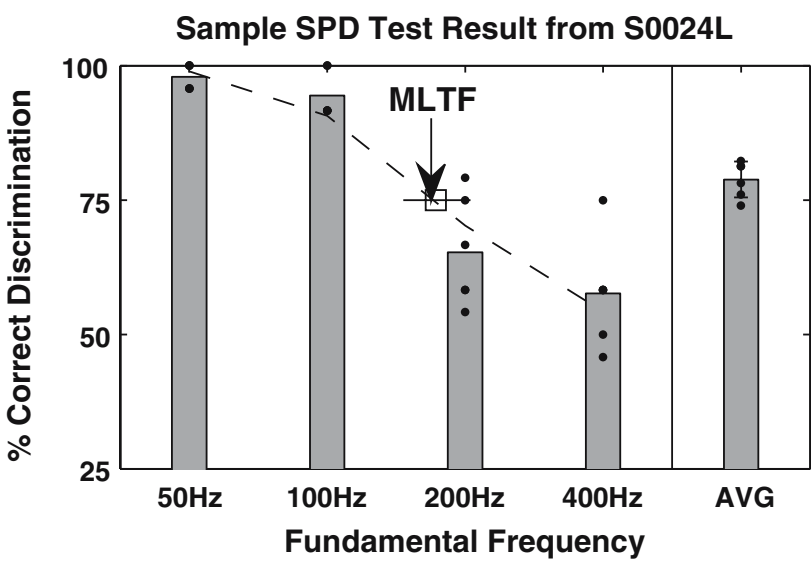

FIG. 2. An example graph demonstrating the six candidate measures of performance on the Schroeder-phase discrimination test. Each filled circle represents a percent correct discrimination for each of the six test blocks. Gray bars represent the AVG across all six test blocks. AVG is the overall score across all fundamental frequencies. The dotted line represents the maximum-likelihood fit of a psychometric curve to the data from the four fundamental frequencies. The open square is the maximum-likelihood predicted $75 \%$ threshold fundamental frequency (MLTF) and the horizontal line is the $95 \%$ confidence interval for the MLTF estimate. prediction of the fundamental frequency at which listeners had a $75 \%$ threshold for discrimination.

\section{General trends}

Visualization of the data for all normal-hearing listeners and CI users in the study reveals some noteworthy trends (Fig. 3). Normal-hearing listeners appear to perform fairly uniformly on this test, with most performing nearly perfectly on all but the highest fundamental frequencies. In contrast, there is a broad range of performance among CI users with some failing to score much above chance (e.g., S0015J and S0034K) and others scoring nearly as well as normal-hearing listeners (e.g., S0000C and S0032A). The population performance worsens with fundamental frequency and the psychometric curve fit appears to approximate the results for many normal-hearing listeners and CI listeners, but some CI users have results that appear nonmonotonic (e.g., S0029D). The appropriateness of assuming a monotonic decrease in performance with fundamental frequency was assessed using a chi-squared test correction for multiple comparisons. Two out of 24 listeners had at least one instance where a score at a higher fundamental frequency was significantly higher $(p<0.01)$ than the score at a lower fundamental frequency. By contrast, 23 out of 24 had at least one low fundamental frequency score that was significantly higher $(p<0.01)$ than a score at high fundamental frequency, showing that, for nearly all CI users, scores do tend to decrease with frequency.

\section{Average $\mathrm{Cl}$ user performance}

Average scores for all CI users are reported in Figure 4 (dark gray bars). The average CI user's performance for $50,100,200$, and $400 \mathrm{~Hz}$ was $84,80,67$, and $58 \%$, respectively. A two-way repeated-measures ANOVA $(4 \times 6$, fundamental frequency by test-block repetitions) revealed a strong effect of fundamental frequency $[F(3,26)=47.9, p<0.001]$, and post hoc analyses revealed that two fundamental frequency pairs $(50$ and $100 \mathrm{~Hz}$ and 200 and $400 \mathrm{~Hz}$ ) were not significantly different from each other, but all other fundamental frequency pairings were significantly different $(p<0.01)$. All of these scores were significantly higher than chance performance on a binomial test with 144 repetitions, suggesting that this fundamental frequency range covers the performance range typical for CI users.

\section{Comparison of normal-hearing listeners and $\mathrm{Cl}$ users}

A comparison between normal-hearing listeners' and CI users' performance is also shown in Figure 4. In 


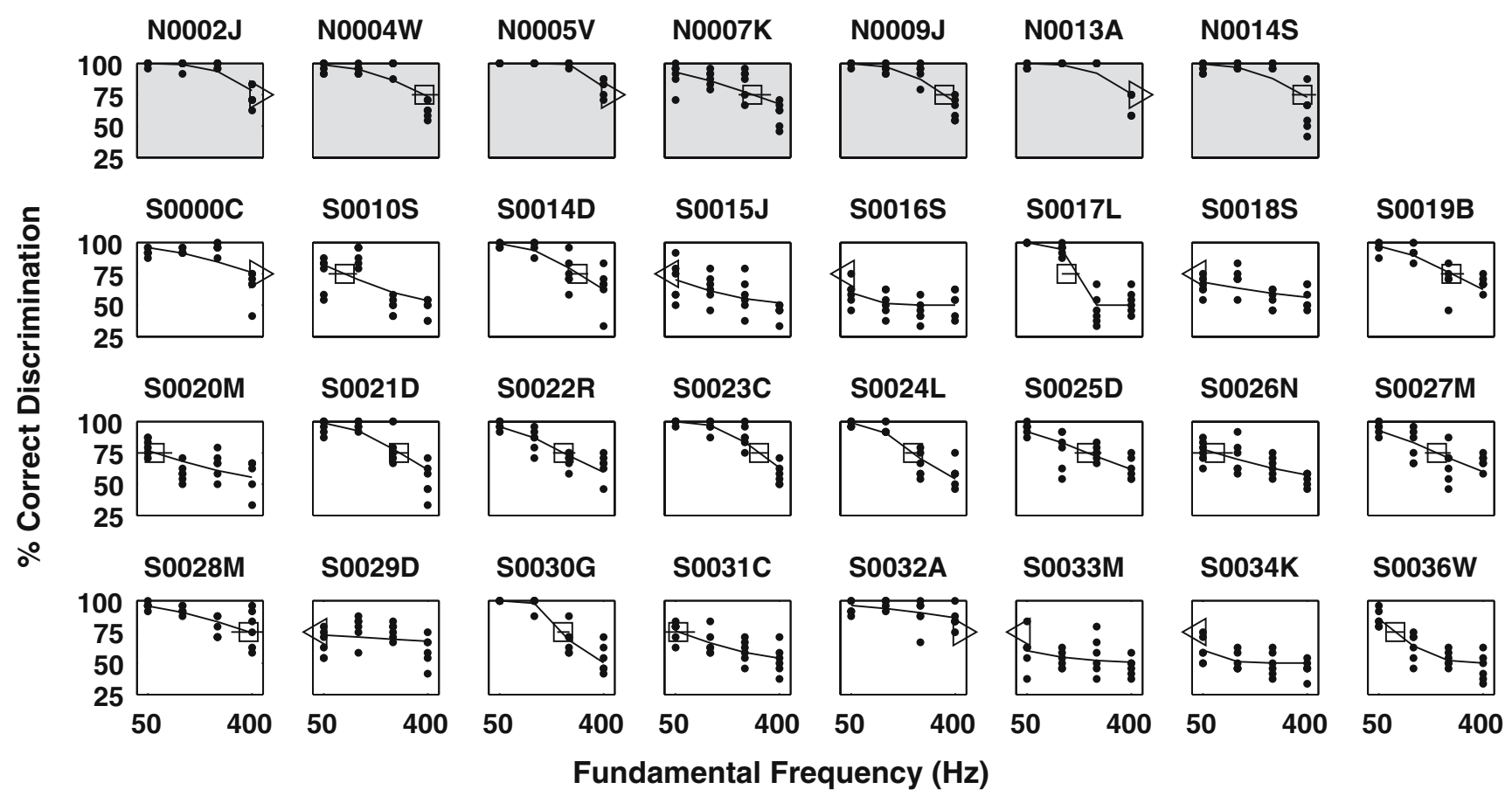

FIG. 3. A summary of data from both normal-hearing listeners (filled gray axes) and $\mathrm{Cl}$ users (white axes) showing scores for each of six test blocks (filled circles) and maximum likelihood fit of a psychometric curve (dotted line). The predicted MLTF (squares) and

contrast to CI users, normal-hearing listeners had 50-, $100-$, 200-, and 400-Hz scores of $97,97,96$, and $66 \%$, respectively, showing a significant decrease in performance at only the highest fundamental frequency tested. Overall performance on the test, the AVG score, was $72 \%$ for CI users, with a range of performances from 51 to $91 \%$, whereas normal-hearing listeners had a mean AVG score of $89 \%$ with a range from 80 to $95 \%$, consistent with the observation that its $95 \%$ confidence interval (solid horizontal line) are shown. Triangles designate that predicted MLTF lie below $50 \mathrm{~Hz}$ (left triangle) or above $400 \mathrm{~Hz}$ (right triangle) for that listener.

the normal-hearing listeners had less variance in their performance on the test. The average predicted threshold fundamental frequency (MLTF) for $75 \%$ correct in normal-hearing listeners was $352 \mathrm{~Hz}$, whereas the average MLTF for CI users was $116 \mathrm{~Hz}$. A nonparametric Kruskall-Wallis test showed that normal-hearing listeners performed significantly better $(p<0.03)$ than CI users for all of the measures of performance. The individual significance values for

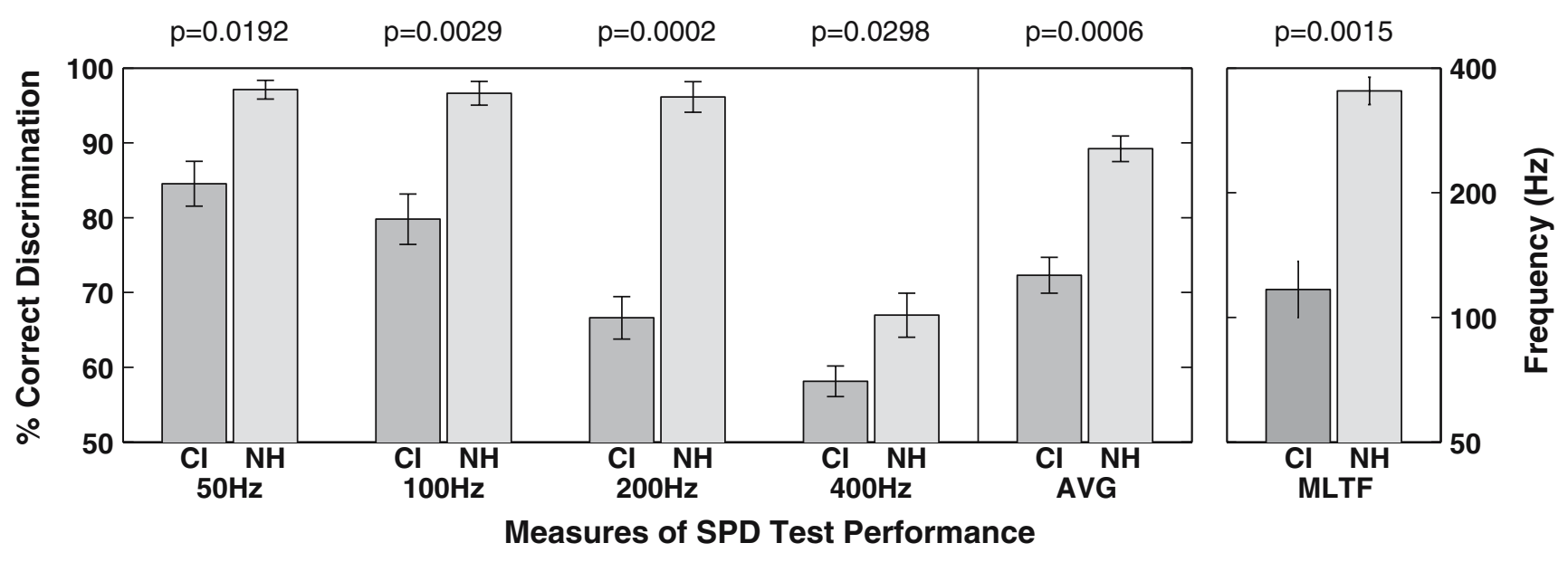

FIG. 4. A comparison of scores on the six measures of Schroederphase discrimination test performance between seven normalhearing listeners $(\mathrm{NH}$, light gray bar) and 24 cochlear implant users (Cl, dark gray bar). The left axis applies to $50-\mathrm{Hz}, 100-\mathrm{Hz}, 200-\mathrm{Hz}$,
400-Hz, and AVG scores and the right axis applies to the maximumlikelihood threshold fundamental frequency (MLTF). $p$ values for a Kruskall-Wallis nonparametric test of significant difference lie above the pair of bars. 


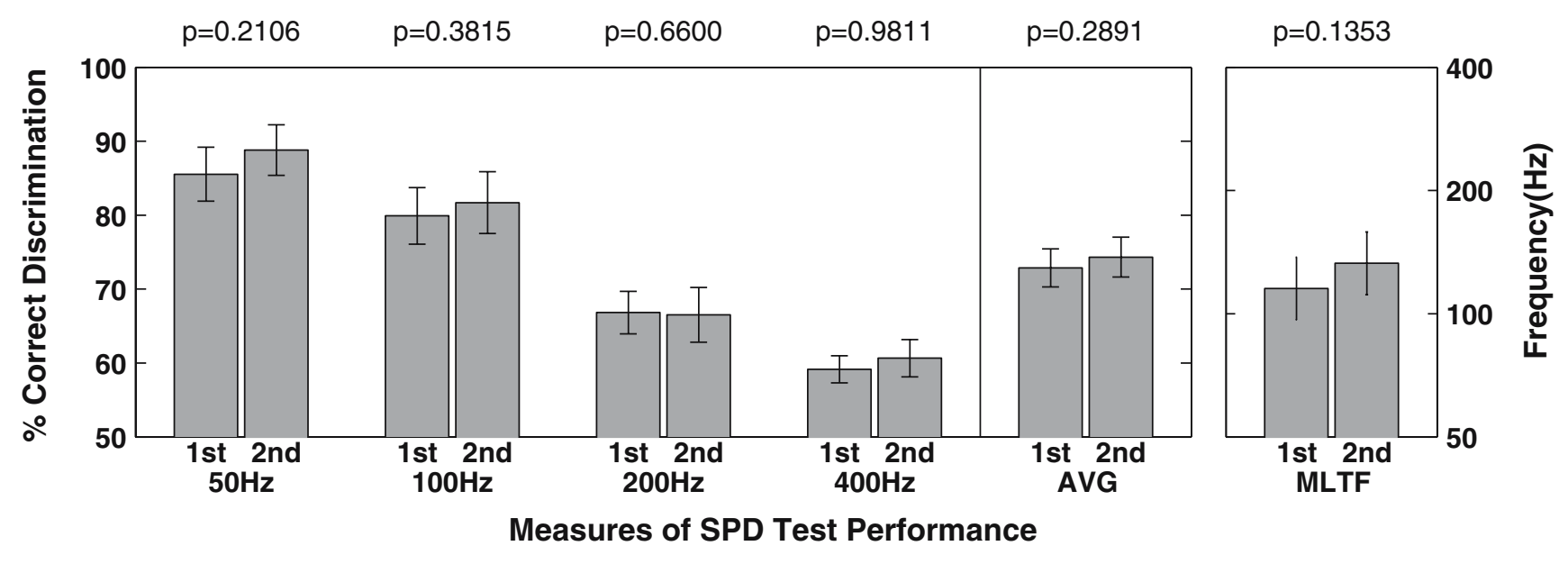

FIG. 5. An analysis of test-retest reliability showing the change in score from first to second test for all six Schroeder-phase discrimination measures. $p$ values for a Wilcoxon Signed-Rank test lie above each bar pair.

these differences are reported above pair of bars in Figure 4.

\section{Learning effects and test-retest reliability}

The $4 \times 6$ (fundamental frequency by test block repetition), two-way repeated-measures ANOVA failed to reveal any significant improvement in scores throughout the six test blocks $[F(5,24)=0.99, p=0.44]$. The test was also repeated by 18 CI users on a second day. Figure 5 shows reliability data with no significant learning effects. Test-retest analysis using the nonparametric Wilcoxon signed-rank test revealed that there was no significant difference between scores the first and second time the test was taken $(p>0.05)$. Thus, test-retest reliability was good. Several (5) of the 18 CI users showed a significant $(p<0.05)$ improvement, whereas only 1 out of 18 had significantly worse performance, suggesting that some minority of CI users might show performance improvement despite no significant learning for the average CI user.

\section{Correlations with demographics and other tests}

The six candidate measures of performance were compared with clinical parameters and performance on other tests. To ensure that both linear and nonlinear relationships between variables were detected, both Pearson's correlation coefficient and Spearman's rank correlation coefficient were computed. Because the Spearman's correlation coefficients did not differ from the Pearson's correlation coefficient in predicting significant correlations, only the Pearson's correlation coefficients are reported. No trend towards correlation (i.e., $p<0.15$ ) was found between age, duration of deafness, duration of implantation, and any of the six measures of performance on the Schroeder-phase discrimination test. Comparisons to other tests were performed for the 20 listeners who completed all of the other tests. No significant correlations were seen between the Schroeder-phase test and the results of the timbre test, the spectral ripple test, or the spondee reception threshold in babble-noise when comparing scores from the first time a listener completed the tests. However, a number of correlations with other tests

TABLE 2

\begin{tabular}{|c|c|c|c|c|c|c|c|c|c|c|c|c|}
\hline \multicolumn{13}{|c|}{ Correlations of Schroeder-phase test measures with other psychophysical tests } \\
\hline & \multicolumn{2}{|l|}{$50 \mathrm{~Hz}$} & \multicolumn{2}{|c|}{$100 \mathrm{~Hz}$} & \multicolumn{2}{|l|}{$200 \mathrm{~Hz}$} & \multicolumn{2}{|l|}{$400 \mathrm{~Hz}$} & \multicolumn{2}{|l|}{$A V G$} & \multicolumn{2}{|l|}{ MLTF } \\
\hline & $R$ & $p$ & $R$ & $p$ & $R$ & $p$ & $R$ & $p$ & $R$ & $p$ & $R$ & $p$ \\
\hline CNC monosyllabic word score & 0.52 & 0.03 & 0.39 & 0.10 & - & - & 0.35 & 0.14 & 0.47 & 0.04 & 0.49 & 0.03 \\
\hline Melody recognition & - & - & 0.38 & 0.10 & - & - & - & - & 0.36 & 0.11 & 0.38 & 0.09 \\
\hline $262 \mathrm{~Hz}$ pitch direction threshold & -0.36 & 0.10 & - & - & -0.37 & 0.10 & -0.53 & 0.02 & -0.39 & 0.08 & -0.35 & 0.12 \\
\hline $330 \mathrm{~Hz}$ pitch direction threshold & - & - & - & - & -0.49 & 0.03 & -0.43 & 0.05 & - & - & - & - \\
\hline Avg. of 4 pitch direction thresholds & - & - & - & - & -0.44 & 0.05 & -0.46 & 0.04 & -0.33 & 0.15 & - & - \\
\hline Spondee reception threshold in steady noise & - & - & - & - & -0.48 & $-\mathbf{0 . 0 3}$ & - & - & - & - & - & - \\
\hline
\end{tabular}

$R$ is the Pearson correlation coefficient, and $p$ is the significance. Bold values have $p<0.05$, italic values have $p<0.15$ 
TABLE 3

Partial correlations controlling for predictive effect of spectral ripple test results

\begin{tabular}{|c|c|c|c|c|c|c|c|c|c|c|c|c|}
\hline & \multicolumn{2}{|l|}{$50 \mathrm{~Hz}$} & \multicolumn{2}{|c|}{$100 \mathrm{~Hz}$} & \multicolumn{2}{|l|}{$200 \mathrm{~Hz}$} & \multicolumn{2}{|l|}{$400 \mathrm{~Hz}$} & \multicolumn{2}{|l|}{$A V G$} & \multicolumn{2}{|c|}{ MILTF } \\
\hline & $R$ & $p$ & $R$ & $p$ & $R$ & $p$ & $R$ & $p$ & $R$ & $p$ & $R$ & $p$ \\
\hline CNC monosyllabic word score & 0.52 & 0.03 & - & - & - & - & - & - & 0.44 & 0.07 & 0.47 & 0.05 \\
\hline Melody recognition & - & - & 0.38 & 0.10 & - & - & - & - & 0.36 & 0.11 & 0.38 & 0.09 \\
\hline $262 \mathrm{~Hz}$ pitch direction threshold & -0.36 & 0.11 & - & - & - & - & -0.53 & 0.02 & 0.36 & 0.12 & - & - \\
\hline $330 \mathrm{~Hz}$ pitch direction threshold & - & - & - & - & -0.44 & 0.05 & -0.49 & 0.03 & - & - & - & - \\
\hline Avg. of 4 pitch direction thresholds & - & - & - & - & -0.37 & 0.11 & -0.52 & 0.02 & - & - & - & - \\
\hline Spondee reception threshold in steady noise & - & - & - & - & -0.48 & 0.03 & - & - & - & - & - & - \\
\hline
\end{tabular}

$R$ is the Pearson correlation coefficient, and $p$ is the significance. Bold values have $p<0.05$, italic values have $p<0.15$

were seen, as summarized in Table 2. Significant $(p<$ $0.05)$ correlations are presented in bold and trends towards correlation $(p<0.15)$ are shown in italics. The results showed that the CNC monosyllabic word score was significantly $(p<0.05)$ correlated with $50-\mathrm{Hz}, \mathrm{AVG}$, and MLTF scores $(R=0.52,0.47,0.49)$, and CNC scores showed a trend toward correlation with the 100- and $400-\mathrm{Hz}$ scores. The melody recognition score showed trends toward correlation with the $100-\mathrm{Hz}, \mathrm{AVG}$, and MLTF scores. The $400-\mathrm{Hz}$ score negatively correlated $(p<0.04)$ with pitch-direction threshold at the $262-\mathrm{Hz}$ base frequency and the average of four pitch-direction thresholds $(R=-0.53,-0.46$, respectively). Finally, the SRT in steady-state speech-shaped noise also significantly correlated with the $200-\mathrm{Hz}$ Schroeder-phase discrimination score $(R=0.48, p<0.03)$.

It has previously been shown that the spectral ripple test correlates significantly with speech understanding in quiet and noise (Henry and Turner 2003; Henry et al. 2005; Won et al. 2007). To determine the extent to which Schroeder-phase discrimination correlates with other tasks independent of spectral resolution, we repeated the above correlation analyses but performed partial correlations controlling for the contribution of the spectral ripple test. This partial correlation analysis (Table 3) produces little change in the results of the correlations from Table 2, modifying $R$ values by no more than 0.07 and modifying $p$ values by no more than 0.05 . None of the significant correlations were strongly affected, suggesting that capacity of the Schroeder-phase measures to predict CNC, speech reception threshold in noise, and pitch-direction threshold was independent of performance on the spectral ripple test.

A complementary analysis was performed to determine if the combination of the spectral ripple test and any of the Schroeder-phase scores could predict performance on music and speech perception tests better than the spectral ripple test or Schroeder-phase scores alone. To be considered a valid, joint contribution to predicting test results, the adjusted $R^{2}$ had to be greater than the $R^{2}$ from the Schroeder-phase or spectral ripple tests, $p$ had to be less than 0.05 , and the regression coefficients had to be nonzero with $95 \%$ confidence for both the spectral ripple test and the Schroeder-phase measures. The combination of the $400-\mathrm{Hz}$ Schroeder-phase discrimination score and the spectral ripple test scores predicted the average pitch-direction threshold for four base frequencies (adjusted $R^{2}=0.42$, whereas $R^{2}=0.29$ and 0.21 for the spectral ripple and $400-\mathrm{Hz}$ scores, respectively). That is, $42 \%$ of the variance for the mean pitch discrimination ability was accounted for by the combination of the 400-Hz Schroeder-phase discrimination score and spectral ripple discrimination. For comparison, this adjusted $R^{2}$ was equivalent to a Pearson correlation coefficient of $R=0.64$.

\section{DISCUSSION}

CI users discriminated positive and negative Schroederphase stimuli above chance but, on average, had significantly poorer performance than normal-hearing listeners at all fundamental frequencies. Like normalhearing listeners, the performance of CI users tended to get worse with increasing fundamental frequency. As noted in the introduction, pulsatile stimulation strategies do not explicitly encode TFS within each channel. Thus, it was expected that CI users would not do as well as normal-hearing listeners on a task intended to measure discrimination of TFS. However, as seen in Figure 1, there are between-channel differences between the positive and negative Schroeder phase, even as processed through a common pulsatile stimulation strategy. In each cycle of positive Schroeder stimuli, the envelope packets sweep downward in frequency across channels through one period. For the negative Schroeder stimuli, the envelope packets sweep upwards over the same period. A correlation analysis of the within- vs. between-channel differences in the $\mathrm{ACE}^{\circledR}$ current-level output for the two Schroeder-phase complexes was completed using a MATLAB Toolbox from Cochlear. Seventy-five percent of the listeners used 
$\mathrm{ACE}^{\circledR}$ processing, and the other types of sound processing were envelope-based, pulsatile strategies. For the within-channel correlation, the correlations between the outputs of single channels for the positiveand negative-phase Schroeder complexes were calculated and averaged. For between-channel correlation, the correlations among the output for all combinations of different channels were determined. The analysis revealed within-channel average correlations of 0.99 for 50-, 200-, and 400-Hz complexes but betweenchannel correlations of $0.53,0.85$, and 0.99 for 50 -, 200-, and 400-Hz Schroeder complexes, respectively. Thus, the between-channel differences in the pulsatile stimulation are much larger than the within-channel differences.

If CI users are using these between-channel differences to discriminate Schroeder-phase complexes, variance in clinical stimulation and processing rates along with the variability in sensitivity to betweenchannel timing differences (Carlyon et al. 2000) could help to explain the wide range of performance seen on this test compared to normal-hearing listeners. At least some CI users are sensitive to phase shifts of pulse trains on pairs of channels when the shifts are on the order of a few milliseconds (Tong and Clark 1986; Carlyon et al. 2000), although it may be necessary that the channels are less than some critical distance apart (McKay and McDermott 1996). This critical distance is likely to vary among listeners, another possible cause for wide variability in the data. Taken as a whole, the correlation analysis and previous results strongly suggest that to discriminate positive and negative Schroeder-phase stimuli, CI listeners use betweenchannel timing differences in the temporal envelopes.

The results of the present study demonstrate that the Schroeder-phase test is a potentially useful measure of performance for CI users. First, the normal-hearing results are consistent with previous studies such as Dooling et al. (2002). Dooling et al.'s change in performance over frequency was similar to the current results, showing a decrease in performance beyond $200 \mathrm{~Hz}$ (see Fig. 5 and Dooling et al. 2002). They used a detection task varying from 0 to $100 \%$ correct performance, but midrange performance was at a similar frequency in both studies. Secondly, the results from CI users typically were above chance and had a broad range, demonstrating utility to evaluate a range of CI users' capabilities. Additionally, test-retest reliability was good. Finally, there was no significant learning trend observed, suggesting that the Schroeder-phase tests are a potentially useful measure of performance over time, or with different sound processing strategies such as in a clinical trial.

Another factor supporting the value of the Schroeder-phase test is that performance on this task correlates significantly with the established CNC- word identification task (Peterson and Lehiste 1962; Thornton and Raffin 1978) for AVG, MLTF, and 50-Hz Schroeder-phase discrimination scores. Evidence suggests that vowel and consonant recognition are correlated with temporal modulation sensitivity (Cazals et al. 1994; Fu 2002). If the Schroeder-phase test is, in part, dependent upon sensitivity to temporal modulations, an association between CNC score and Schroederphase measures would be expected. Consonant-vowel transitions require sensitivity to a dynamic spectral change, much like Schroeder-phase discrimination requires sensitivity to a dynamic spectral change. The period of the $50-\mathrm{Hz}$ stimuli is $20 \mathrm{~ms}$. The duration of a typical consonant-vowel transition is about $40 \mathrm{~ms}$ (Kewley-Port et al. 1983). The better the temporal resolution is, the better the listener will be able to hear the trajectory of the transition. A resolution of $5 \mathrm{~ms}$, for example, would yield good information for the dynamic changes in the spectral profile for consonant-vowel transition, as well as for the $50-\mathrm{Hz}$ Schroeder-phase stimuli. -Discriminating a dynamic spectral change might also require some spectral resolution; thus, spectral resolution was evaluated and a partial correlation was done controlling for the effects of spectral resolution. With this control, the correlation between the $50-\mathrm{Hz}$ Schroeder stimulus and CNC words was exactly the same (0.52), suggesting that the underlying element of Schroeder-phase discrimination accounting for $27 \%$ of the variance in CNC words was a temporal element.

Modest correlations were also found among the $200-\mathrm{Hz}$ Schroeder tests and speech perception in steady-state noise. We speculate that the $200-\mathrm{Hz} /$ speech-in-noise relationship might be related to the ability of the CI users to segregate out the target female speech (which has an approximately $200-\mathrm{Hz}$ fundamental) from the steady-state noise. The target speech would have modulation frequencies near the speaker's fundamental frequency, about $200 \mathrm{~Hz}$.

Correlations were found between $400-\mathrm{Hz}$ Schroeder and pitch-direction discrimination at $362-\mathrm{Hz}$ fundamental and between the $400-\mathrm{Hz}$ Schroeder score and the average pitch-direction discrimination thresholds for all fundamental frequencies tested. The correlation between the $400-\mathrm{Hz}$ score and pitch-direction threshold might be explained with a between-channel theory. In the pitch-direction test, users were asked to discriminate complex tones. Complex tones of differing fundamental frequency generate unique spectral profiles that are more discernible with increased spectral resolution. However, harmonics falling within the same frequency band lead to temporal modulations beating at the fundamental frequency. Therefore, perception of the pitch of harmonic complexes might reasonably be correlated with temporal modulation and, as a result, the Schroeder-phase discrimination test measures. Based on the spectral and temporal theories of 
pitch perception (Wightman and Green 1974), pitch discrimination could be improved by improving either temporal or spectral resolution. Consistent with these theories, pitch-direction thresholds in CI users were predicted jointly (adjusted $R^{2}=0.42$, equivalent $R=$ 0.64 ) by the Schroeder-phase test and the spectral ripple tests. In this case of CI users, the temporal information would be transmitted via temporal envelope modulations rather than TFS.

Finally, no correlation was found between understanding speech in a two-speaker babble background and Schroeder phase. As noted in the introduction, the task of identifying speech in fluctuating backgrounds requires the ability to discern TFS (Qin and Oxenham 2003; Füllgrabe et al. 2006). The mechanisms required to do well on Schroeder-phase discrimination are apparently not the same as the mechanisms required to do well on speech discrimination in fluctuating backgrounds. Presumably, the speech-in-babble task requires sensitivity to within-channel TFS cues to help segregate the fundamental frequencies of different voices. We speculate that if new processing schemes delivered within-channel information, performance on the Schroeder-phase discrimination would improve and a correlation between speech understanding in fluctuating backgrounds and Schroeder-phase discrimination would be observed.

In conclusion, the present study has demonstrated that, although most CI sound processing algorithms do not explicitly encode TFS, Schroeder-phase discrimination is still possible, given sufficient between-channel differences in temporal modulations. Schroeder-phase discrimination ability at $50-\mathrm{Hz}$ and AVG performance over all fundamental frequencies has also been shown to correlate with word recognition, and at $200 \mathrm{~Hz}$, Schroeder-phase discrimination was correlated with the ability to understand a female speaker in steady-state, speech-shaped noise. Furthermore, 200- and 400-Hz Schroeder-phase discrimination ability was correlated with pitch-direction discrimination ability using complex tones with similar fundamental frequencies. These correlations were independent of spectral ripple discrimination ability, suggesting independent spectral and temporal processes underlying complex-tone pitch perception in CI listeners.

\section{ACKNOWLEDGEMENTS}

We appreciate the dedicated efforts of our listeners. The authors also thank Robert Kang, Jong Ho Won, Grace Nimmons, and Jonathan Kopelovich for their help in administering the psychophysical tests. Nikita Imenov, Leah Drennan, Jong Ho Won, and two anonymous reviewers provided helpful comments on previous versions of this manuscript. The study was supported by NIH grants T32-
GM07266, R01-DC007525, and P30-DC04661 and Cochlear Corporation.

\section{REFERENCES}

Carlyon RP, Geurts L, Wouters J. Detection of small across-channel timing differences by cochlear implantees. Hear. Res. 141:140154, 2000.

Cazals Y, Pelizzone M, Saudan O, Boex C. Low-pass filtering in amplitude modulation detection associated with vowel and consonant identification in subjects with cochlear implants. J. Acoust. Soc. Am. 96:2048-2054, 1994.

Dooling RJ, Leek MR, Gleich O, Dent ML. Auditory temporal resolution in birds: discrimination of harmonic complexes. J. Acoust. Soc. Am. 112:748-759, 2002.

DrEnNAN WR, RubinsteIn JT. Sound processors in cochlear implants. In: Waltzmann SB and Roland JT (eds) Cochlear Implants. New York, NY, Thieme, pp. 40-47, 2006.

DrEnNAN WR, WONJH, DasikaVK, RUBINSTEINJT. Effects of temporal fine structure on the lateralization of speech and on speech understanding in noise. J. Assoc. Res. Otolaryngol. 8:384-392, 2007.

Fu Q-J. Temporal processing and speech recognition in cochlear implant users. Neuroreport 13:1635-1639, 2002.

Füllgrabe C, Berthommier F, Lorenzi C. Masking release for consonant features in temporally fluctuating background noise. Hear. Res. 211:74-84, 2006.

Gfeller K, Woodworth G, Robin DA, Witt S, Knutson JF. Perception of rhythmic and sequential pitch patterns by normally hearing adults and adult cochlear implant users. Ear Hear. 18:1-15, 1997.

Gfeller K, Turner C, Mehr M, Woodworth G, Fearn R, Knutson JF, WitT S, Stordahl J. Recognition of familiar melodies by adult cochlear implant recipients and normal-hearing adults. Cochlear Implants Int. 3:31-55, 2002.

Gfeller K, Olsqewski C, Rychener M, Sena K, Knutson JF, Witt S, MacPherson B. Recognition of "Real-world" musical excepts by cochlear implant recipients and normal-hearing adults. Ear Hear. 26:237-250, 2005.

GHitzA O. On the upper cutoff frequency of the auditory criticalband envelope detectors in the context of speech perception. J. Acoust. Soc. Am. 110:1628-1640, 2001.

Gilbert G, Lorenzi C. The ability of listeners to use recovered envelope cues from speech fine structure. J. Acoust. Soc. Am. 119:2438-2444, 2006.

Henry BA, Turner CW. The resolution of complex spectral patterns by cochlear implant and normal-hearing listeners. J. Acoust. Soc. Am. 113:2861-2873, 2003.

Henry BA, Turner CW, Behrens A. Spectral peak resolution and speech recognition in quiet: normal hearing, hearing impaired, and cochlear implant listeners. J. Acoust. Soc. Am. 118:1111$1121,2005$.

Johnson DH. The relationship between spike rate and synchrony in responses of auditory-nerve fibers to single tones. J. Acoust. Soc. Am. 68:1115-1122, 1980.

Kewley-Port D, Pisoni DB, Studdert-Kennedy M. Perception of static and dynamic acoustic cues to place of articulation in initial stop consonants. J. Acoust. Soc. Am. 73:1779-1793, 1983.

Kohlrausch A, Sander A. Phase effects in masking related to dispersion in the inner ear. II. Masking period patterns of short targets. J. Acoust. Soc. Am. 97:1817-1829, 1995.

Kong Y-Y, Cruz R, Jones JA, Zeng F-G. Music perception with temporal cues in acoustic and electric hearing. Ear Hear. 25:173-185, 2004.

Kubli LR, Leek MR, Dreisbach LE. Acoustic reflexes to Schroederphase harmonic complexes in normal-hearing and hearingimpaired individuals. Hear. Res. 202:1-12, 2005. 
LEVITT H. Transformed up-down methods in psychoacoustics. J. Acoust. Soc. Am. 49:467-477, 1971.

LoIzou P. Mimicking the human ear: an overview of signalprocessing strategies for converting sound into electrical signals in cochlear implants. IEEE Signal Process. Mag. 98:101-130, 1998.

Madigan R, Williams D. Maximum-likelihood psychometric procedures in two-alternative forced-choice: evaluation and recommendations. Percept Psychophys 42:240-249, 1987.

McKay CM, McDermott HJ. The perception of temporal patterns for electrical stimulation presented at one or two intracochlear sites. J. Acoust. Soc. Am. 100:1081-1092, 1996.

Nelson PB, Jin S-H, Carney AE, Nelson DA. Understanding speech in modulated interference: cochlear implant users and normalhearing listeners. J. Acoust. Soc. Am. 113:961-968, 2003.

Nimmons G, Kang R, Drennan W, Longnion J, Ruffin C, Worman T, Yueh B, Rubinstein J. Clinical assessment of music perception in cochlear implant listeners. Otol Neurotol DOI 10.1097/mao. 0b013e31812f7244, 2007.

Peterson GE, Lehiste I. Revised CNC lists for auditory tests. J. Speech Hear. Disord. 27:62-70, 1962.

Qin MK, Oxenham AJ. Effects of simulated cochlear-implant processing on speech reception in fluctuating maskers. J. Acoust. Soc. Am. 114:446-454, 2003.

Rosen S. Temporal information in speech: acoustic, auditory and linguistic aspects. Philos. Trans. R. Soc. Lond. B Biol. Sci. 336:367-373, 1992.

SCHROEDER MR. Synthesis of low-peak-factor signals and binary sequences with low autocorrelation. IEEE Trans. Inf. Theory 16:85-89, 1970

Senn P, Kompis M, Vischer M, Haeusler R. Minimum audible angle, just noticeable interaural differences and speech intelligibility with bilateral cochlear implants using clinical speech processors. Audiol Neurootol. 10:342-352, 2005.

SHANNon R. Temporal modulation transfer functions in patients with cochlear implants. J. Acoust. Soc. Am. 91:2156-2164, 1992.

Summers V. Effects of hearing impairment and presentation level on masking period patterns for Schroeder-phase harmonic complexes. J. Acoust. Soc. Am. 108:2307-2317, 2000.
Summers V, Leek MR. Masking of tones and speech by Schroederphase harmonic complexes in normally hearing and hearingimpaired listeners. Hear. Res. 118:139-150, 1998.

Summers V, de Boer E, Nuttall AL. Basilar-membrane responses to multicomponent (Schroeder-phase) signals: understanding intensity effects. J. Acoust. Soc. Am. 114:294-306, 2003.

Thornton AR, Raffin MJM. Speech-discrimination scores modeled as a binomial variable. J. Speech Hear. Res. 21:507-518, 1978.

Tong YC, Clark GM. Loudness summation, masking, and temporal interaction for sensations produced by electric stimulation of two sites in the human cochlea. J. Acoust. Soc. Am. 79:1958-1966, 1986.

Townshend B, Cotter N, Compernolle DV, White RL. Pitch perception by cochlear implant subjects. J. Acoust. Soc. Am. 82:106-115, 1987.

Turner CW, Gantz BJ, Vidal C, Behrens A, Henry BA. Speech recognition in noise for cochlear implant listeners: benefits of residual acoustic hearing. J. Acoust. Soc. Am. 115:1729-1735, 2004.

Venzon D, Moolgavkar S. A method for computing profile-likelihoodbased confidence intervals. Appl. Stat. 37:87-94, 1988.

Wightman FL, Green DM. The perception of pitch. Am. Sci. 62:208215, 1974.

WiLSON BS. Engineering design of cochlear implants. In: Zeng F-G, Popper A, and Fay RR (eds) Cochlear Implants: Auditory Prostheses and Electric Hearing. New York, Springer-Verlag, pp. 14-52, 2004

Won JH, Drennan WR, Rubinstein JT. Spectral-ripple resolution correlates with speech reception in noise in cochlear implant users. J. Assoc. Res. Otolarygol. 8:384-392, 2007.

ZeNG F-G. Temporal pitch in electric hearing. Hear. Res. 174:101106, 2002.

ZeNG FG. Auditory prostheses: past, present, and future. In: Zeng FG, Popper AN, and Fay RR (eds) Cochlear Implants: Auditory Prostheses and Electric Hearing. New York, Springer-Verlag, pp. 1-13, 2004.

Zeng FG, Nie K, Liu S, Stickney G, Rio ED, Kong YY, Chen H. On the dichotomy in auditory perception between temporal envelope and fine structure cues. J. Acoust. Soc. Am. 116:1351-1354, 2004.

Zeng F-G, Nie K, Stickney GS, Kong Y-Y, Vongphoe M, Bhargave A, WEI C, CAO K. Speech recognition with amplitude and frequency modulations. Proc. Natl. Acad. Sci. U. S. A. 102:2293-2298, 2005. 